This item was submitted to Loughborough's Institutional Repository (https://dspace.lboro.ac.uk/) by the author and is made available under the following Creative Commons Licence conditions.

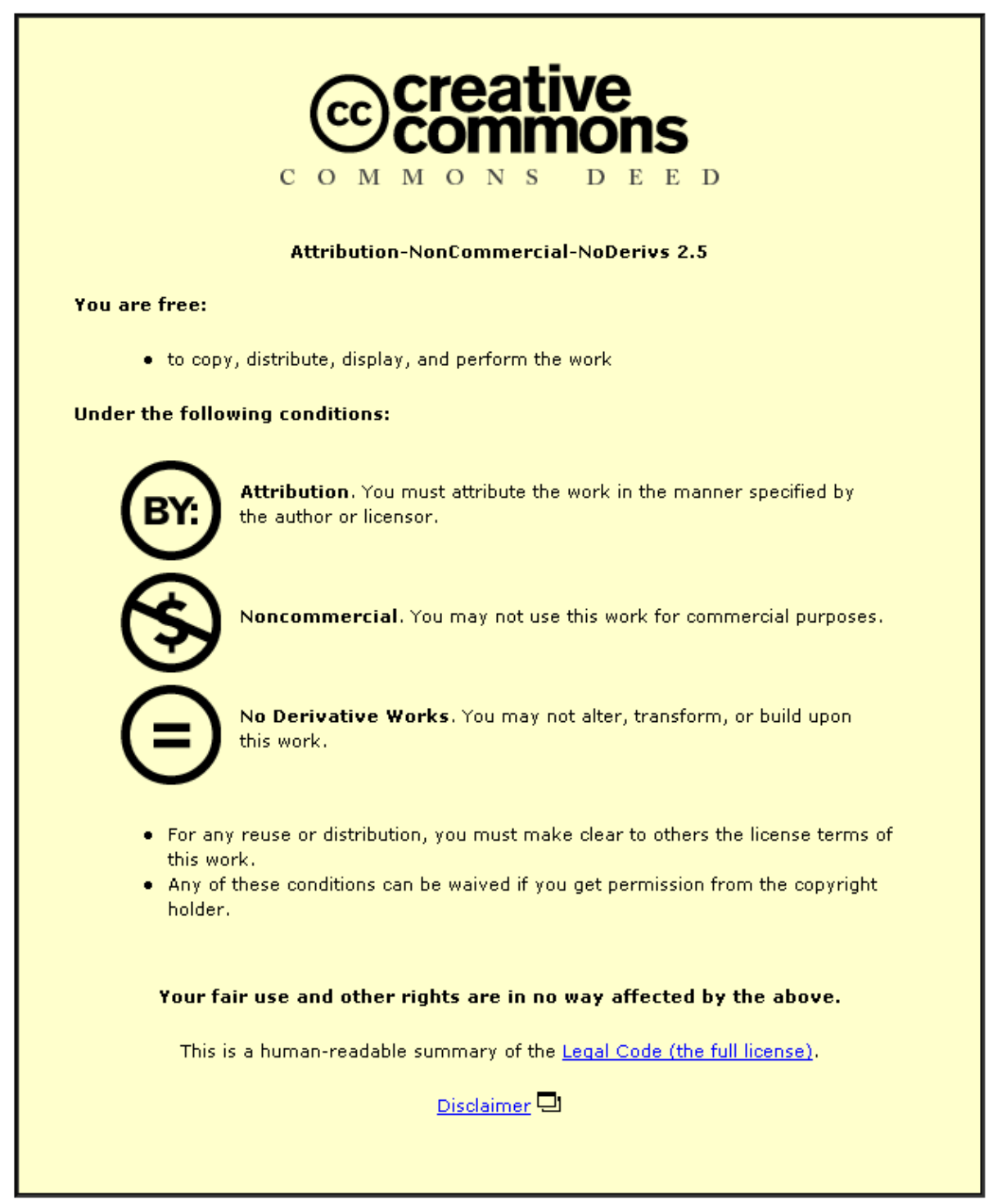

For the full text of this licence, please go to: http://creativecommons.org/licenses/by-nc-nd/2.5/ 


\title{
An experimental analysis of the effects of noise on Wi-Fi video streaming
}

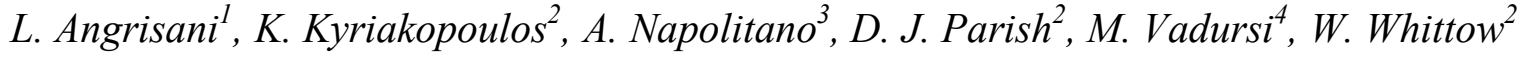 \\ ${ }^{1}$ Dept. of Computer Science and Control Systems, University of Naples Federico II, Via Claudio 21, I-80125 Napoli \\ Phone : +390817683170 - Fax : +390817683816-Email : angrisan@unina.it \\ ${ }^{2}$ Dept. of Electronic and Electrical Engineering, Loughborough University, Loughborough, LE11 3TU, U.K. \\ Email: \{elkk; elwgw; d.j.parish\}@lboro.ac.uk \\ ${ }^{3}$ Dept. of Electrical Engineering, University of Naples Federico II, Via Claudio 21, I-80125 Napoli \\ Phone : +390817683866-Fax : +390812396897-Email : aniello.napolitano@unina.it \\ ${ }^{4}$ Dept. of Technologies, University of Naples "Parthenope", Centro Direzionale di Napoli Isola C4, I-80143 Napoli Phone : \\ +390815476791 - Fax : +390815474777 - Email : michele.vadursi@uniparthenope.it
}

\begin{abstract}
Wireless networks such as WiFi suffer communication performance issues in addition to those seen on wired networks due to the characteristics of the radio communication channel used by their Physical Layers (PHY). Understanding these issues is a complex but necessary task given the importance of wireless networks for the transfer of wide ranging packet steams including video as well as traditional data. Simulators are not accurate enough to allow all the intricacies of such communication to be accurately understood, especially when complex interactions between the protocols of different layers occurs. The paper suggests cross layer measurement as a solution to the problem of understanding and analysis of such complex communication issues and proposes a framework in which appropriate performance measurements can be made from a WiFi network supporting a video streaming application. The framework has been used to collect these measurements at the PHY, MAC, Transport and Application layers. Analysis of the collected measurements has allowed the effects of noise interference at the PHY to be related to the perceived performance at the Application Layer for a video streaming application. This has allowed the effect of the SNR on the download time of a video sequence to be studied.
\end{abstract}

Keywords: Cross-layer measurements, Video streaming, Wireless networks, $\mathrm{Wi}-\mathrm{Fi}$.

\section{INTRODUCTION}

Wireless Local Area Networks (WLANs) compliant with the IEEE 802.11 standard (Wi-Fi networks) are nowadays widely spread, thanks to their easiness of installation, low cost and great flexibility. They allow for wireless access to the Internet and network connectivity. Hotspots for Wi-Fi connections are present almost everywhere: in offices, factories homes, airports, schools, universities, commercial centers and even parks. A great interest for Wi-Fi applications is also rapidly growing in the field of real-time multimedia, for applications such as audio, video streaming, and videosurveillance over a wireless link.
Despite such a huge development, the performance of $\mathrm{Wi}$ Fi networks is sometimes hard to predict and to guarantee. This is mainly due to the poor stability and reliability of the radio link. In fact, while on wired channels signal integrity is assured by mechanical, electrical and protocol characteristics of the physical and data link layers, on wireless channels unpredictable and uncontrollable interference can severely affect data transmission, and ultimately degrade or even compromise the desired performance of the network [1]. The Wi-Fi standard exploits a scarce, shared, and noisy spectrum, i.e. the unlicensed $2.4 \mathrm{GHz}$ Industrial Scientific Medical (ISM) band, on which other devices may operate simultaneously [2$6]$.

Performance evaluation based on simulations can be of help, but is not sufficient, due to the great number of variables involved $[7,8]$. In such a direction, useful information can be achieved through ad-hoc laboratory and on-field measurements, exploiting proper test beds. In the recent past, cross-layer measurements have come out to be a powerful option to assess and predict the performance of wireless and hybrid networks, as well as to troubleshoot them [9-14]. In the literature, a number of papers investigate on the feasibility of video streaming over Wi-Fi networks. In many cases, efficient solutions are proposed for improving the quality of video streaming. Nevertheless, only few of such contributions face the problem from an experimental point of view $[15,16]$.

This paper presents a test bed and a measurement method to experimentally analyze the effects of noise on real $\mathrm{Wi}$-Fi video streaming applications. The paper presents the method, the test bed, and the first experimental results. The ultimate goal is to understand how and in which ways noise at the physical layer can affect the streaming time of video in the application layer of Wi-Fi networks.

\section{PROPOSED METHOD}

Tests are conducted within a protected and controlled environment, i.e. a shielded semi-anechoic chamber compliant with electromagnetic compatibility requirements for radiated 
emission tests. Experiments aim to emulate the actual operating scenario of a WLAN compliant with IEEE 802.11g standard. In particular, two different measurement scenarios are accounted for. In the first scenario a noise generator aims at the $\mathrm{AP}$ and in the second, aims at the client side. For each scenario, a cross-layer approach is applied in order to assess the performance of WLAN in supporting video streaming applications. More specifically, different metrics at different protocol stack layers are measured, such as streaming time at application layer, packet retransmission and lost packets at transport layer, MAC retransmission at link layer and SNR level at physical layer at both server $\left(\mathrm{SNR}_{\mathrm{AP}}\right)$ and client side $\left(\mathrm{SNR}_{\mathrm{C}}\right)$.

The adopted test-bed presented in Fig.1, entails the following components:

1) a WAP54G access point (AP) by Linksys, compliant with the IEEE $802.11 \mathrm{~g}$ standard;

2) a notebook "Host2", used as client, equipped with Intel Core 2 Duo@2 GHz processor, running Mac Os X and Linux through virtualisation, 4 GB RAM and built-in Wi-Fi card (Airport) compliant with IEEE 802.11b/g standard;

3) a notebook "Host1", used as server, with Intel Pentium Dual Core@1.73 GHz,running Windows XP, 1 GB of RAM

4) an ASUS USB adapter Wi-Fi card, namely WL-167G, compliant with IEEE $802.11 \mathrm{~g}$ standard;

5) an arbitrary waveform generator, namely Agilent Technologies ESG E4438C $\mathrm{CM}^{\mathrm{TM}}(250 \mathrm{kHz}-6 \mathrm{GHz}$ output frequency range), with arbitrary waveform generation capability ( $80 \mathrm{MHz}$ modulation bandwidth, 16 bit vertical resolution, 8 Megasample memory depth), which is used to generate white Gaussian noise;

6) a microwave directive broad-band horn antenna Schwarzbeck BBHA9120D (1 - $18 \mathrm{GHz}$ frequency range).

Host1 operates outside the semi-anechoic chamber and communicates with the AP at a $100 \mathrm{Mbps}$ rate and through a 5 m length UTP category 3 cable. On the other hand, Host 2 and AP use a wireless connection on channel 6 (i.e. $2.437 \mathrm{GHz}$ carrier frequency), according to a DCF MAC layer access method along with CSMA/CA protocol, and operate at fixed positions, represented by white and gray squares in Fig. 1.

Their mutual distance is referred to as $r$ in the figure. The horn antenna is located in-between the AP and Host2, thus at a distance of nearly equal to $r / 2$ from both AP and Host 2 and aiming the AP initially and later the client. In this way, when aiming at the AP, interfering phenomena, due to the noise levels, are principally located at the AP side. In contrast, Host2 does not suffer from their effects as the horn is a highly directive antenna. Similarly, when aiming Host2, interfering phenomena, are principally located at the client side.

Generally, Host 2 generates a request to Host1, through the AP by wireless connection, for streaming an mpeg-4 encoded video located on Host 1 . Once the connection is accepted, Host1 sends the video to Host2 via the AP, exploiting TCP connection. Host 2 streams the mpeg- 4 video using the iTunes application. Several video transmissions are executed from
Host 1 to Host 2 for different noise levels and for each test the cited metrics are measured at different layers.

To this aim, suitable sniffer software, namely Wireshark [17] is installed on both video transmitter and receiver in order to measure both TCP packet retransmissions and losses, as well as the streaming time at application layer. An Asus Wi-Fi card, connected to Host 2 and operating in monitor mode, is exploited to gather the packets retransmission at MAC layer. Otherwise, they could not be captured from the machine that is generating the retransmissions neither from the machine that the retransmissions are destined to. However, due to the different polarization of the Asus card, not all frames seen by the client's built-in Airport card could be seen by Asus.

It is crucial to understand that each monitoring point has its own vantage point (VP) and should be treated as an independent witness. Each measurement point can reveal issues that would be otherwise hidden by examining just the other two measurement points.

Once the network configuration has been fixed, a preliminary measurement procedure is carried out, with an omnidirectional broad-band antenna POD $16(1-18 \mathrm{GHz}$ frequency range) connected to a real time spectrum analyzer, namely Tektronix RSA3408 (1 - $6 \mathrm{GHz}$ frequency range), in order to measure SNR levels at both AP and the client. In particular, the useful signal power inside channel 6 is measured keeping only the WLAN connection on and locating the POD 16 antenna alternately next to AP and STA, for measuring respectively the signal power radiated both by the client to AP $\left(\mathrm{P}_{\mathrm{AP}}\right)$ and by AP to the client $\left(\mathrm{P}_{\mathrm{C}}\right)$.

To this aim, the antenna is oriented so as to be polarized according to the AP and the client, respectively. Note, that while measuring the signal from client at the AP position, the AP was temporarily moved to the far end of the chamber and shielded from the POD antenna by radar absorbing material in order to avoid measuring the AP's power signal.

Afterwards, the generator is switched on and emits white Gaussian noise in channel 6; the noise power level is then measured after switching off the WLAN connection, while maintaining the POD16 in the same positions as in the previous step. In this way, the noise power received at AP $\left(\mathrm{N}_{\mathrm{AP}}\right)$ and client $\left(\mathrm{N}_{\mathrm{C}}\right)$ is suitably evaluated. The measured values of useful and noise power levels, for different noise levels, are finally used to determine the SNR at both sides, respectively expressed by equations (1) and (2).

Subsequently, after measuring the SNR values, the video streaming is started and all the data needed to evaluate important metrics at network, transmission and application layer (e.g. transport layer retransmissions, lost segments, streaming time, number of broken connections, number of lost packets, one way delay, etc.) are logged and successively analyzed.

$$
\begin{aligned}
& \mathrm{SNR}_{\mathrm{AP}}=\mathrm{P}_{\mathrm{AP}} / \mathrm{N}_{\mathrm{AP}} \\
& \mathrm{SNR}_{\mathrm{C}}=\mathrm{P}_{\mathrm{C}} / \mathrm{N}_{\mathrm{C}}
\end{aligned}
$$




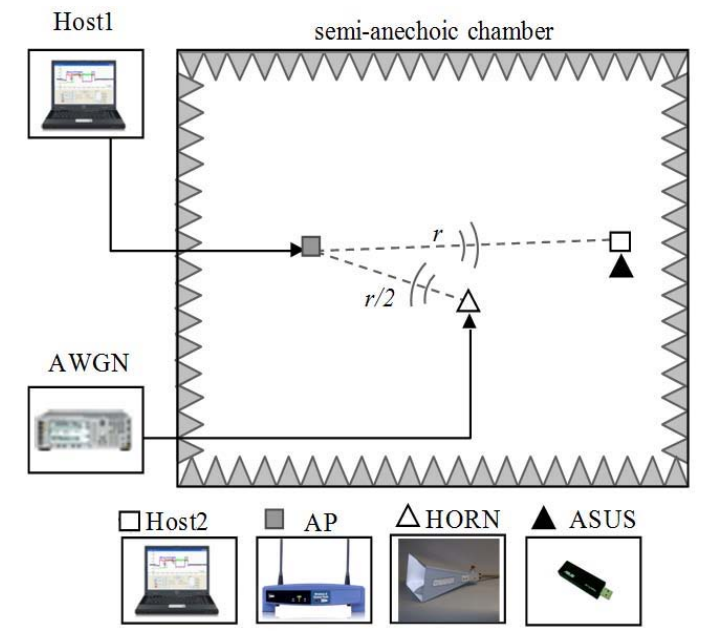

Figure 1. First measurement scenario, adopted test-bed

\section{RESULTS}

Initially, experiments were carried out by switching off the noise and verifying that the WLAN succeeds in transfering the video clip without packet loss. Successively, the noise level has been increased until the wireless connection between the AP and the client is dropped. When aiming the AP, it is worth noting that $\mathrm{SNR}_{\mathrm{C}}$ doesn't change when different noise values are imposed on the generator, because the horn antenna is aiming at the AP and the noise at the client is negligible. Similarly, when aiming the client, $\mathrm{SNR}_{\mathrm{AP}}$ is constant.

Table 1, shows the results obtained for different $\mathrm{SNR}_{\mathrm{AP}}$ values. The following conclusions can be made.

- $\quad \mathrm{An}_{\mathrm{SNR}} \mathrm{AP}$ level below $27 \mathrm{~dB}$ causes a breakdown of the wireless connection: the wireless connection is initially on, and most packets are transmitted, but after some minutes the communication suddenly disrupts giving rise to freezing phenomenon on the video clip.

- The streaming time abruptly increases with increased noise up to a value of $145.3 \mathrm{~s}$ for an $\mathrm{SNR}_{\mathrm{AP}}$ equal to $27 \mathrm{~dB}$.

- $\quad \mathrm{An}_{\mathrm{SNR}} \mathrm{AP}$ equal to $32 \mathrm{~dB}$ grants no TCP retransmission even though many MAC packets are retransmitted. This phenomenon is due to the MAC mechanism of the IEEE $802.11 \mathrm{~b} / \mathrm{g}$ standard, which is based on a classic CSMA/CA. After transmitting each packet, the MAC layer at the server side attempts to send the next packet only after a layer-2 acknowledgment from the client has arrived. At the client side, if the packet is correctly received, the layer- 2 acknowledgment is sent to the server and, once successfully arrived, it is moved up to the transport layer; otherwise, the client waits for a retransmission. This way, the number of retransmissions at MAC layer is not necessarily correlated to the number of TCP Retransmissions; in fact, it can be much higher.

- $\quad$ For $\mathrm{SNR}_{\mathrm{AP}}=29.5 \mathrm{~dB}$, the statistics TCP lost segments and

TCP Retransmissions (ReTx) are equal to three. The three TCP lost segments reported by Wireshark are not real, but an error of the underlying software/hardware. From the monitoring log files obtained from the client, the reported TCP lost segments have different sequence number. However, the TCP ReTx refer to the same segment being retransmitted three times. These 3 TCP ReTx have no relation with the three TCP lost segments.

The error in the number of TCP lost segments happens because Wireshark could not record all packets received and wrongly assumes that some TCP segments were missing. This is not the case, as from the log files of the server it is corroborated that the client has sent acknowledgments for the "supposed" lost segments. In addition, if indeed the segments were lost, then the server should have retransmitted them, which is not the case as can be seen from the server's log files.

We proceed by examining why three retransmissions were generated for a particular TCP segment. Figure 2 shows the occurrence of the original TCP segment and its three retransmissions as reported by both the server and the client. The client's last TCP ACK packet expects this segment (as shown from the server's log file). As seen in Fig. 2 the TCP segment is transmitted three times but the next ACK that covers this particular TCP segment is not received by the server. As a result, streaming stops there.

The observation is that by aiming the Horn antenna at the AP, packets destined to the AP have difficulty arriving correctly. On the other hand, judging by the unnecessary three retransmissions from the AP and their successful arrival at the client, it seems there is no problem for packets going the other direction. In other words the frames from the client have difficulty being received by the antenna of the AP when subject to noise.

This observation can be also corroborated by comparing the number of TCP Duplicate (Dup) ACKs sent from the client (Asus VP) and the number of TCP Dup ACKs actually received at the server (Table 1).

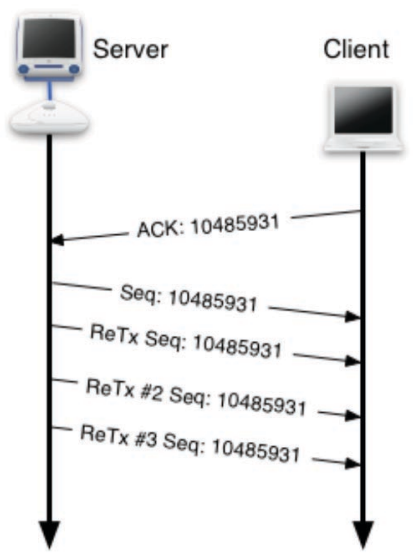

Figure 2. A schematic of packet flow, indicating that when directing noise at the AP, frames can be transmitted but TCP ACKs can not be received by AP

Table 2 shows the measured statistics when aiming the Horn antenna at the client side. The statistic TCP ReTx from the client VP does not really represent how many TCP segments have been retransmitted from the server. Only the transmitter knows how many times has retransmitted TCP segments. These are much more than what the client perceives (due to losses in the channel - see Table 2). Thus, there seems to be a problem for frames transmitted from the AP to enter 
the noisy area around the client. Therefore, the high number of TCP lost segments from client VP and high number of TCP ReTx from the server VP.

On the other hand, frames from client (ACKs usually) do not have a particular problem traveling from the noisy area to the AP. The high number of TCP Dup ACKs is actually due to multiple requests for retransmission of TCP segments that the client has not received or received out of order.

If the TCP server receives 3 Dup ACKs for the same segment, it takes this as an indication that the next segment has been lost. Because the server sends a large number of segments back to back, if one segment is lost, there will likely be many back-to-back duplicate ACKs. In our case, the server received more than 100 requests for a particular segment [18].

A possible reason for this phenomenon is that the radio receiver cannot "track" the pre-amble of the 802.11 frames due to the noise in the surrounding environment and due to the deterioration of the signal as it travels from one side to the other. Previous work has shown that the MAC layer modulation is robust enough against noise environments and high enough noise power is required to corrupt frames [M].

Figure 3 shows the relation between the streaming time of the video and the SNR conditions for both scenarios: horn aiming the AP and horn aiming the client. The behavior of both lines is similar with a knee determining the sudden deterioration of the performance. For the AP the knee happens at around $32 \mathrm{~dB}$ and for the client at around $24 \mathrm{~dB}$. Therefore, in this case, the client is more robust to noise.

Therefore, when streaming TCP based video, the streaming time (and hence user-perceived performance) is influenced by the SNR at both the server and the client ends. This is because TCP operation requires the successful receipt of acknowledgements at the server in order to continue data streaming. As such, noise at both ends of the connection influences the performance.

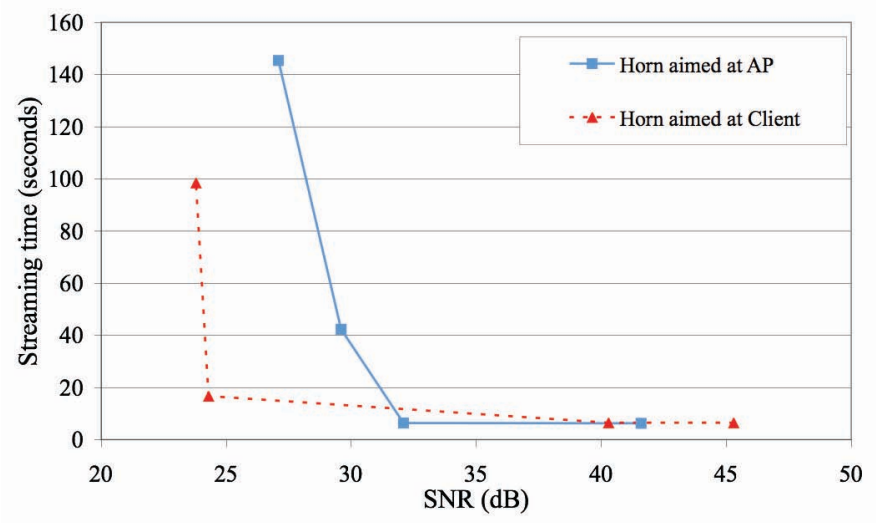

Figure 3. Streaming time versus SNR

\section{CONCLUSIONS - FUTURE WORK}

This paper has presented a cross layer measurement framework for a Wi-Fi network when carrying streamed traffic such as video downloads. The measurements produced from an implementation of the framework have allowed details of the interactions of the protocols at different layers of the network operation to be analysed in detail. In particular, some of the characteristics of the retransmission processes at both the MAC and the Transport (TCP) layers have been clearly seen. The relationship between the SNRs at the client and server ends of the connection and the download time has also been examined using the framework. The measurement approach is also applicable to other wireless and combined wireless/wired network technologies and for the detailed understanding of the cross layer characteristics pertinent to other applications.

As for future work, the relationships between streaming times and SNR are not very complex and could be captured in a model in order to predict application performance which would be a function of both $\mathrm{SNR}_{\mathrm{AP}}$ and $\mathrm{SNR}_{\mathrm{C}}$.

\section{REFERENCES}

[1] A. P. Jardosh, K. N. Ramachandran, K. C. Almeroth, E. M. BeldingRoyer, "Understanding Congestion in IEEE $802.11 \mathrm{~b}$ Wireless Networks", Proc. of Internet Measurement Conference IMC 2005, pp. 279-292.

[2] M. van der Schaar, Y. Andreopoulos, and Z. Hu, "Optimized scalable video streaming over IEEE $802.11 \mathrm{a} / \mathrm{e}$ HCCA wireless networks under delay constraints," IEEE Trans. Mobile Comput., vol. 5, no. 6, pp. 755768, June 2006.

[3] D. Majumdar, G. Sachs, I.V. Kozintsev, and K. Ramchandran, "Multicast and Unicast Real-Time Video Streaming over Wireless LANs," IEEE Trans. Circuits and Systems for Video Technology, vol. 12, no. 6, pp. 524-534, June 2002.

[4] J. Kuri and S.K. Kasera, "Reliable multicast in multi-access wireless LANs", Wireless Networks, Vol. 7, No. 4, pp. 359-369, July 2001.

[5] I. Haratcherev, J. T. K. Langendoen, R. Lagendijk, and H. Sips, "Optimized Video Streaming over 802.11 by Cross-Layer Signaling", IEEE Communications Magazine, January 2006.

[6] K. Balachandran, S. R. Kadaba, and S. Nanda, "Channel Quality Estimation and Rate Adaptation for Cellular Mobile Radio," IEEE $J S A C$, vol. 17, no. 7, July 1999, pp. 1244-56.

[7] D. Moltchanov, Y. Koucheryavy, J. Harju, "Cross-layer analytical modeling of wireless channels for accurate performance evaluation," Proc. of QoFiS'2004, Barcelona, Spain, Oct. 2004, pp. 194-203.

[8] S. Pollin, B. Bougard. G. Lenoir, B. Van Poucke, L. Van der Perre, F. Catthoor, I. Moerman "Cross-layer exploration of link adaptation in wireless LANs with TCP traffic," IEEE Symposium on Communications and Vehicular Technology (SCVT'03), Eindhoven (NL), Nov. 2003.

[9] L. Angrisani, A. Pescapè, G. Ventre, M. Vadursi, "Performance measurement of IEEE 802.11b-based networks affected by narrowband interference through cross-layer measurements," IET Communications (formerly IEE Proceedings Communications), vol. 2, No.1, Gennaio 2008, pp. 82-91.

[10] L. Angrisani, M. Vadursi, “Cross-Layer Measurements for a Comprehensive Characterization of Wireless Networks in the Presence of Interference," IEEE Trans. on Instrumentation and Measurement, vol.56, No.4, Aug. 2007, pp.1148-1156.

[11] K. G. Kyriakopoulos, W. Whittow, D. J. Parish, "A Framework for Cross-Layer Measurements in Wireless Networks", Proc. of the Fifth IEEE Advanced Inter-national Conference on Telecommunications (AICT 2009) part of WebTel 2009, Venice, Italy, 24-28 May 2009. 
[12] R. Li, D. J. Parish, K. G. Kyriakopoulos, "A Framework for Cross-layer Measurement of 3G and Wi-Fi Combined Networks", Proc. of PGNet 2008: The $9^{\text {th }}$ Annual Post Graduate Symposium on The Convergence of Telecommunications, Networking \& Broadcasting, Liverpool, U.K., 2324 June 2008.

[13] A. Sheth, C. Doerr, D. Grunwald, R. Han, D. Sicker, "MOJO: A Distributed Physical Layer Anomaly Detection System for 802.11 WLANs," Proc. of MobiSys'06, June 19-22, 2006, pp. 191-204.

[14] J. Yeo, M. Youssef, T. Henderson, A. Agrawala, "An Accurate Technique for Measuring the Wireless Side of Wireless Networks," Proc of the Intern. Workshop on Wireless traffic measurements and modelling WiTMeMo 2005, pp. 13-18.

[15] L. Angrisani, A. Napolitano, A. Sona, "Are IEEE 802.11 Wireless Networks Appropriate for Video Streaming in the Presence of
Interference? An Experimental Answer," Proc. of $I^{2}$ MTC 2008, IEEE Int. Instr. and Meas. Tech. Conf., Victoria, Canada, May 12-15, 2008 pp. $1722-1727$

[16] L. Angrisani, M. Bertocco, A. Gamba, A. Sona, "Modeling the Performance of CSMA-CA Based Wireless Networks versus Interference Level," Proc. of $I^{2}$ MTC 2008, IEEE Int. Instr. and Meas. Tech. Conf., Victoria, Canada, May 12-15, 2008 pp.376-381.

[17] http://www.wireshark.org [online].

[18] James F. Kurose and Keith W. Ross, "Computer Networking: A TopDown Approach Featuring the Internet”, Addison Wesley, 2003

TABLE I. CROSS-LAYER Metrics WHEN THE HoRn AiMED AT THE AP

\begin{tabular}{|c|c|c|c|c|}
\hline & \multicolumn{4}{|c|}{$\mathrm{SNR}_{\mathrm{AP}}(\mathrm{dBm})$} \\
\hline & 32 & 29.5 & 27 & 22 \\
\hline TCP ReTx from Server (Client VP) & 0 & 3 & 0 & 38 \\
\hline TCP Lost segments from Server (Client VP) & 4 & 3 & 6 & 7 \\
\hline TCP Dup ACKs from client (Asus VP) & 121 & 8107 & 29584 & 20091 \\
\hline TCP Dup ACKs from client (Server VP) & 0 & 0 & 3 & 156 \\
\hline MAC ReTx frames from Server (Asus VP) & 187 & 192 & 396 & 3033 \\
\hline Streaming time (s) & 6.4 & 92.9 & 145.3 & 164.1 \\
\hline Video freezing effect / Video finishes ? & No/Yes & Yes/No & Yes/Yes & Yes / No \\
\hline
\end{tabular}

TABLE II. CROSS-LAYER METRICS WHEN THE HORN AIMED AT THE CLIENT

\begin{tabular}{|c|c|c|c|c|c|}
\hline & \multicolumn{5}{|c|}{ SNR $_{\mathrm{C}}(\mathrm{dBm})$} \\
\hline & No noise & $\mathbf{- 4 5 . 3}$ & $\mathbf{- 4 0 . 3}$ & $\mathbf{- 2 4 . 3}$ & $\mathbf{- 2 3 . 8}$ \\
\hline TCP ReTx from Server (Server VP) & 1 & 0 & 0 & $\mathbf{1 0 8}$ & $\mathbf{3 8 7}$ \\
\hline TCP Lost segments from Server (client VP) & 5 & 9 & 6 & 118 & 378 \\
\hline TCP ReTx from Server (client VP) & 0 & 0 & 0 & $\mathbf{1 7}$ & $\mathbf{1 5 8}$ \\
\hline TCP Duplicate ACKs from client (Server VP) & 0 & 0 & 0 & $\mathbf{2 0 5 4}$ & $\mathbf{3 5 6 6}$ \\
\hline TCP Fast ReTx from Server (Server VP) & 0 & 0 & 0 & $\mathbf{9 0}$ & $\mathbf{2 9 8}$ \\
\hline MAC ReTx frames from Server (Asus VP) & 131 & 163 & 212 & 141 & 7514 \\
\hline Streaming time (s) & 6.39 & 6.54 & 6.47 & 16.76 & 98.48 \\
\hline Video freezing effect/Video finishes ? & No/Yes & No/Yes & No/Yes & No/Yes & No/Yes \\
\hline
\end{tabular}

